\title{
Influence of Glutathione S-Transferase B (Ligandin) on the Intermembrane Transfer of Bilirubin

\author{
Implications for the Intracellular Transport of Nonsubstrate Ligands in Hepatocytes
}

Stephen D. Zucker, ${ }^{\star}$ Wolfram Goessling, ${ }^{*}$ Bernard J. Ransil, ${ }^{\star}$ and John L. Gollan*

*Division of Gastroenterology, Brigham and Women's Hospital, ${ }^{\ddagger}$ Charles A. Dana Research Institute, Harvard-Thorndike Laboratory and Department of Medicine, Beth Israel Hospital, Harvard Medical School and Harvard Digestive Diseases Center, Boston, Massachusetts 02115

\begin{abstract}
To examine the hypothesis that glutathione S-transferases (GST) play an important role in the hepatocellular transport of hydrophobic organic anions, the kinetics of the spontaneous transfer of unconjugated bilirubin between membrane vesicles and rat liver glutathione S-transferase B (ligandin) was studied, using stopped-flow fluorometry. Bilirubin transfer from glutathione $S$-transferase $B$ to phosphatidylcholine vesicles was best described by a single exponential function, with a rate constant of $8.0 \pm 0.7 \mathrm{~s}^{-1}( \pm \mathrm{SD})$ at $25^{\circ} \mathrm{C}$. The variations in transfer rate with respect to acceptor phospholipid concentration provide strong evidence for aqueous diffusion of free bilirubin. This finding was verified using rhodamine-labeled microsomal membranes as acceptors. Bilirubin transfer from phospholipid vesicles to GST also exhibited diffusional kinetics. Thermodynamic parameters for bilirubin dissociation from GST were similar to those for human serum albumin. The rate of bilirubin transfer from rat liver basolateral plasma membranes to acceptor vesicles in the presence of glutathione $S$-transferase B declined asymptotically with increasing GST concentration. These data suggest that glutathione S-transferase B does not function as an intracellular bilirubin transporter, although expression of this protein may serve to regulate the delivery of bilirubin, and other nonsubstrate ligands, to sites of metabolism within the cell. (J. Clin. Invest. 1995. 96:1927-1935.) Key words: bilirubin - glutathione transferases • biological transport • kinetics • intracellular membranes
\end{abstract}

Preliminary reports of this work have been published in abstract form (1993. Gastroenterology. 104:1025a and 1995. Gastroenterology. 108:1073a).

Address correspondence to Stephen D. Zucker, M.D., Division of Gastroenterology, Brigham \& Women's Hospital, 75 Francis Street, Boston, MA 02115. Phone: 617-732-5828; FAX: 617-730-5807.

Received for publication 16 March 1995 and accepted in revised form 6 July 1995

1. Abbreviations used in this paper: bLPM, basolateral plasma membrane; CDNB, 1-chloro-2,4-dinitrobenzene; dansyl-PE, $N$-(5-dimethylaminonaphthalene-1-sulfonyl ) dipalmitoyl-L- $\alpha$-phosphatidylethanolamine; FABP, fatty acid binding protein; GST, glutathione S-transferases; rhod-PE, ( $N$-lissamine rhodamine B sulfonyl)diacyl-L$\alpha$-phosphatidylethanolamine.

J. Clin. Invest.

(c) The American Society for Clinical Investigation, Inc.

0021-9738/95/10/1927/09 \$2.00

Volume 96, October 1995, 1927-1935

\section{Introduction}

The glutathione S-transferases (GST) ${ }^{1}$ represent an important family of detoxifying isoenzymes, which catalyze the conjugation of glutathione to hydrophobic substrates that bear an electrophilic functional group $(1,2)$. In addition to this enzymatic function, GST also exhibit high-affinity binding of a variety of endogenous and xenobiotic substances (2-6), which do not undergo metabolism (nonsubstrate ligands). One such compound, unconjugated bilirubin ( bilirubin-IX $\alpha$ ), the hydrophobic end product of heme degradation, is bound efficiently by a member of the alpha class of GST, glutathione S-transferase B (ligandin) $(3,7-10)$. This protein, which consists of homoand heterodimers of Ya and Yc subunits (2), is present in high concentration in the liver, kidney, intestine, and adrenal (11), and represents the principal intracellular binding protein for bilirubin in both rat and human liver $(3,12,13)$. Indeed, the hepatocyte is a major site of GST expression, with glutathione $\mathrm{S}$-transferase B constituting as much as $5 \%$ of total soluble protein $(14,15)$. The other classes of GST ( $m u$ and $p i$ ) exhibit unique tissue distributions and are distinguished from alpha class proteins on the basis of substrate specificity, affinity for nonsubstrate ligands, and isoelectric point (2).

It has long been postulated that GST serve a cellular transport function, carrying hydrophobic nonsubstrate ligands through the cytosol to intracellular sites of metabolism $(1,4$, $6,13,16)$. While refuted by some authors $(17,18)$, there is evidence to suggest that GST facilitate the movement of heme out of mitochondria $(19,20)$, and GST YbYb ( $m u$ class) has been implicated in the transport of hormones to the cell nucleus $(6,21)$. The mechanism by which the GST are postulated to enhance the rate of intracellular diffusion is via an increase in the effective aqueous concentration of the ligand (13). However, to date, there has been little direct evidence to support this hypothesis. In fact, the GST exhibit several physical properties which are atypical for intracellular transport proteins. For instance, the molecular masses of the various GST (44-50 kD) are two- to fourfold higher than those of other well characterized transporters, such as fatty acid binding proteins (22), nonspecific lipid transfer protein (23), and phosphatidylcholine transfer protein (23). The relative large size of the GST would result in a reduced rate of intracellular diffusion $(24,25)$, an attribute which is poorly suited to efficient transport function. Moreover, GST undergo dimerization, a characteristic which has not been identified in other intracellular transport proteins. Finally, GST possess an essential enzymatic function which is independent of the transported (nonsubstrate) ligand, a property not otherwise observed with other transporters. Thus, there are many unusual features of the GST which cast doubt on their capability to effectively function as cytosolic transport proteins. 


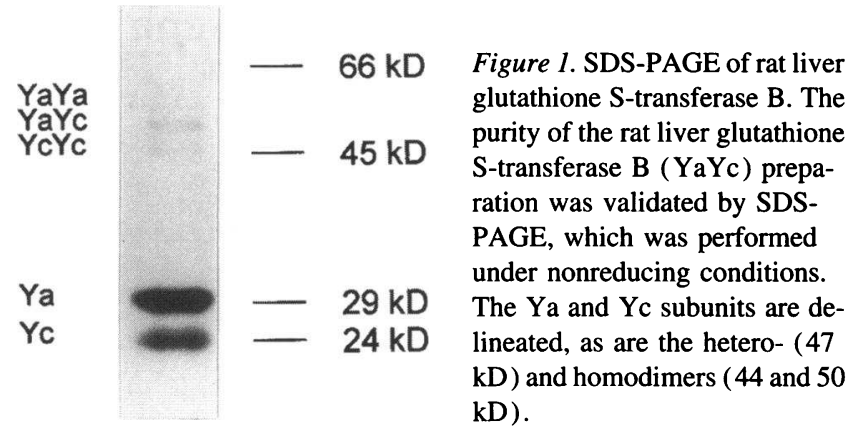

We have demonstrated previously that the spontaneous transfer of bilirubin between small unilamellar phosphatidylcholine vesicles is rapid, with a halftime of $\sim 3 \mathrm{~ms}(26)$, and that the lipid composition of intracellular membranes may facilitate the trafficking of bilirubin from the plasma membrane to the endoplasmic reticulum of the hepatocyte (27). Based on these findings, we have postulated that the hepatocellular transport of bilirubin is mediated, at least in part, by cellular membranes. Our investigations were designed to elucidate the kinetic mechanisms of bilirubin binding to and dissociation from glutathione S-transferase B, and to directly examine the effect of this protein on the rate of intermembrane bilirubin transfer. Contrary to generally held concepts regarding the role of GST in the intracellular transport of nonsubstrate ligands, the results of these studies suggest that glutathione S-transferase B does not facilitate the aqueous movement of bilirubin.

\section{Methods}

Materials. Egg lecithin (grade 1) used in the preparation of phospholipid vesicles was obtained from Lipid Products (Surrey, United Kingdom). The fluorescent phospholipid probes $N$-(5-dimethylaminonaphthalene-1-sulfonyl) dipalmitoyl-L- $\alpha$-phosphatidylethanolamine (dansyl$\mathrm{PE})$ and ( $N$-lissamine rhodamine B sulfonyl) diacyl-L- $\alpha$-phosphatidylethanolamine (rhod-PE) were purchased from Avanti Polar Lipids (Birmingham, AL). Rat liver glutathione S-transferase B (YaYc fraction) was obtained from Biotrin International (Dublin, Ireland) and was further characterized by SDS-PAGE (Fig. 1). Bilirubin-IX $\alpha$ was purchased from Porphyrin Products, Inc. (Logan, UT), and purity was documented at $>99 \%$ by absorbance in chloroform solution $\left(\epsilon_{453}\right.$ $=62,000 \mathrm{M}^{-1} \cdot \mathrm{cm}^{-1}$ ) and by reverse phase HPLC (28). Unfractionated rat liver GST, 1-chloro-2,4-dinitrobenzene (CDNB), and reduced glutathione (GSH) were purchased from Sigma Chemical Company (St. Louis, MO). All glassware was chloroform washed to avoid potential lipid contamination.

Assay of GST activity. GST activity was determined using the method of Habig and Jakoby (29). Measurements were performed by incubating $1.0 \mathrm{mM}$ CDNB and $1.0 \mathrm{mM}$ GSH in $0.1 \mathrm{M}$ potassium phosphate ( $\mathrm{pH} 6.5)$ at $25^{\circ} \mathrm{C}$ and measuring spectrophotometric changes at $340 \mathrm{~nm}$ after the addition of an amount of enzyme which resulted in an absorbance change of $<0.05 / \mathrm{min}$ over $3 \mathrm{~min}$. All activities were corrected for nonenzymatic rates. The specific activity of rat liver glutathione S-transferase B (YaYc) was $11 \mu \mathrm{mol} \cdot \mathrm{min}^{-1} \cdot \mathrm{mg}^{-1}$, consistent with reported values (29). Unfractionated GST exhibited a specific activity of $27 \mu \mathrm{mol} \cdot \mathrm{min}^{-1} \cdot \mathrm{mg}^{-1}$. This elevated value likely reflects the presence of glutathione $S$-transferase $A\left(\mathrm{Yb}_{1} \mathrm{Yb}_{1}\right)$ in the preparation, which exhibits a significantly higher activity against CDNB as compared with the B isoform (29).

Preparation of small unilamellar vesicles and incorporation of fuorescent probes. Small unilamellar vesicles were prepared by a modification (26) of the sonication procedure of Barenholz et al. (30). Phos- pholipids were solubilized in ether, evaporated under argon atmosphere, and then desiccated overnight under vacuum. The dried phospholipids were suspended in aqueous buffer and sonicated on ice under an argon atmosphere, followed by centrifugation to sediment large vesicle contaminants. Vesicle phospholipid concentration was quantified using the lipid phosphorus assay of Bartlett (31).

Isolation and fluorescent labeling of microsomal membrane vesicles from rat liver. Rat liver microsomal membranes were isolated from fasted male Sprague-Dawley rats (Charles River Breeding Laboratories, Inc., Wilmington, MA), as described previously (27). Livers were resected and homogenized in $0.25 \mathrm{M}$ sucrose, $10 \mathrm{mM}$ Tris- $\mathrm{HCl}, 1 \mathrm{mM}$ $\mathrm{MgCl}_{2}$ ( $\mathrm{pH} 7.3$ ), followed by serial centrifugation at $41,000 g_{\text {ave }}$ for 10 min and 80,000 $g_{\text {ave }}$ for $25 \mathrm{~min}$. The phospholipid probe, rhod-PE, was incorporated into microsomal membranes at a concentration of $1 \mathrm{~mol} \%$ phospholipid, according to the method of Stubbs et al. (32). A chloroform solution of rhod-PE was evaporated under argon and desiccated overnight. Freshly prepared microsomes were added to the dried lipids ( $5.6 \mu \mathrm{g}$ rhod-PE/mg microsomal protein), which were resuspended and, after a 10-min incubation at room temperature, centrifuged at 80,000 $g_{a v e}$ for $25 \mathrm{~min}$. The labeled microsomal membranes were harvested, stored at $4^{\circ} \mathrm{C}$, and used within $6 \mathrm{~h}$ of preparation. Protein was quantified by the Bio-Rad assay (Bio-Rad Laboratories, Hercules, CA).

Isolation of rat basolateral liver plasma membranes. Hepatocyte basolateral liver plasma membranes (bLPM) were prepared using the method of Meier and Boyer (33), as described previously (27). The livers of male Sprague-Dawley rats were excised, homogenized, centrifuged for $15 \mathrm{~min}\left(1,500 \mathrm{~g}_{\text {ave }}\right)$, and the pooled pellets were subjected to sucrose density centrifugation $\left(90,000 g_{\text {ave }}\right)$ for $90 \mathrm{~min}$ in a swinging bucket rotor. Mixed liver plasma membranes were harvested, washed twice $\left(2,700 \mathrm{~g}_{\text {ave }}\right.$ for $\left.15 \mathrm{~min}\right)$, rehomogenized, and subjected to repeat sucrose density centrifugation $\left(197,500 g_{\text {ave }}\right)$ for $3 \mathrm{~h}$. The basolateral membrane fraction was harvested, sedimented $\left(115,000 g_{\text {ave }}\right.$ for 60 min), and resuspended in $0.25 \mathrm{M}$ sucrose, $0.2 \mathrm{mM} \mathrm{CaCl}, 5 \mathrm{mM}$ $\mathrm{MgSO}_{4}$, and $20 \mathrm{mM}$ Hepes/Tris (pH 7.4) buffer. Membranes were stored at $-70^{\circ} \mathrm{C}$ and used within 2 wk of preparation. The enrichment of the bLPM preparation (35-40-fold) was determined by the coupled kinetic assay for ouabain-sensitive $\mathrm{Na}^{+} / \mathrm{K}^{+}$-ATPase and $\mathrm{Mg}^{2+}$-ATPase, as described by Scharschmidt and colleagues (34).

Measurement of bilirubin transfer from glutathione $S$-transferase $B$ to acceptor vesicles. The spontaneous transfer of bilirubin from rat liver glutathione S-transferase B ( YaYc) to acceptor phospholipid vesicles was monitored by changes in the intrinsic tryptophan fluorescence of GST induced by bilirubin binding $(5,10)$. Since unconjugated bilirubin is insoluble at neutral pH (35), incorporation into GST (or phospholipid vesicles) was accomplished by dissolving the bile pigment in alkaline buffer, followed by rapid neutralization $(26,36)$. A fluorometer (Applied Photophysics, Leatherhead, United Kingdom) equipped with an SPF-17 stopped flow device was used to monitor the rate of reemergence of protein fluorescence (excitation [ex ]: $280 \mathrm{~nm}$, emission [em] : 320 $\mathrm{nm}$ long-pass filter) as bilirubin transferred from glutathione S-transferase B (1:1 molar ratio) to small unilamellar phosphatidylcholine acceptor vesicles (26). At these excitation and emission wavelengths, the contribution of bilirubin fluorescence (37) to the total signal was negligible $(<0.1 \%)$. In an attempt to simulate intracellular conditions, transfer experiments were conducted in a $0.1 \mathrm{M} \mathrm{KCl}, 10 \mathrm{mM}$ Tris- $\mathrm{HCl}$, $1 \mathrm{mM}$ EDTA buffer solution ( $\mathrm{pH} 7.4$ ), and all steps were performed in the dark to minimize bilirubin photodegradation.

As native hepatocyte membranes exhibit substantial tryptophan fluorescence (27), the rate of bilirubin transfer from glutathione S-transferase $\mathrm{B}$ to membrane vesicles also was determined by labeling acceptor membranes with a fluorescent phospholipid and monitoring changes in probe fluorescence. Initially, a model system was used in which dansyl$\mathrm{PE}$ was incorporated into small unilamellar phosphatidylcholine vesicles at a concentration of $0.2 \mathrm{~mol} \%$ (36). When a solution of GST-bound bilirubin is mixed with these labeled vesicles, quenching of dansyl fluorescence (ex: $340 \mathrm{~nm}$, em: $520 \mathrm{~nm}$ long-pass filter) is observed as the bilirubin transfers from the protein to the acceptor vesicles. Using a similar experimental approach, the rate of bilirubin transfer from gluta- 
thione S-transferase B to rhodamine-labeled microsomal membrane vesicles also was measured. Bilirubin, when excited at $450 \mathrm{~nm}$, transfers energy to the rhodamine probe on binding to labeled microsomal vesicles (em: $535 \mathrm{~nm}$ long-pass filter), thereby enabling bilirubin transfer from GST to be monitored by the time-dependent increase in rhodamine fluorescence.

Determination of the rate of bilirubin transfer from small unilamellar phosphatidylcholine vesicles to GST. The rate of bilirubin transfer from small unilamellar phosphatidylcholine donor vesicles (containing $0.5 \mathrm{~mol} \%$ dansyl-PE) to GST was determined using stopped-flow fluorometry. After the rapid mixing of GST with bilirubin-incorporated (quenched) vesicles, reemergence of dansyl fluorescence (ex: $340 \mathrm{~nm}$, em: $520 \mathrm{~nm}$ long-pass filter) was observed as bilirubin transferred from the donor vesicles to the protein acceptor. Unfractionated (total) rat liver GST, as opposed to the YaYc fraction, were used in these experiments due to the prohibitive protein concentrations required.

Measurement of bilirubin transfer from basolateral plasma membranes to acceptor vesicles in the presence of glutathione S-transferase $B$. Studies of bilirubin transfer from rat basolateral liver plasma membranes to small unilamellar phosphatidylcholine vesicles labeled with $0.2 \mathrm{~mol} \%$ dansyl-PE were performed as outlined in the preceding sections. Dansyl-labeled acceptor vesicles were combined with varying concentrations of glutathione S-transferase B before stopped-flow mixing with bilirubin-loaded bLPM donor vesicles. The rate of appearance of bilirubin at the acceptor vesicles was determined by monitoring the quenching of dansyl fluorescence.

Kinetic analysis of bilirubin transfer data. The time course for bilirubin transfer was analyzed by fitting the time-dependent changes in fluorescence intensity to both single and double exponential functions, with fit quality assessed by ANOVA $(26,27)$. The pattern of variation in the bilirubin transfer rate with respect to the concentration of the donor and the acceptor facilitates the distinction between a collisional and a diffusional mechanism of transfer (38), as previously demonstrated for the interaction of bilirubin with serum albumin (36). Initial experiments used a two component system to examine the mechanism of bilirubin transfer between GST and membrane vesicles (39). Most intracellular transfer proteins exhibit collisional ligand transfer kinetics $(38,40-43)$ such that, during the transfer process, the protein forms a transient complex with the donor or acceptor membrane. For a collisional mechanism of bilirubin transfer between glutathione S-transferase B and acceptor vesicles, the overall transfer rate can be expressed as (36):

$R_{\text {coll }}=\frac{K_{a}^{G S T}[G S T]+K_{a}^{V}[V]}{\frac{1}{k_{\text {off }}^{G S T}}+\frac{1}{K_{\text {off }}^{V}}}$

where $[G S T]$ is the concentration of glutathione S-transferase B, $[V]$ is the acceptor vesicle phospholipid concentration, and $K_{a}^{G S T}$ and $K_{a}^{v}$ are the association constants for GST with the bilirubin-vesicle complex and vesicles with the bilirubin-GST complex, respectively. The dissociation rate constants for GST and vesicles from the GST-bilirubinvesicle complex are indicated by $k_{\text {off }}$ with superscript parameters.

For a diffusional transfer mechanism, ligand interaction with acceptor membranes is presumed to occur after dissociation from GST and diffusion through the aqueous phase, with the transfer rate given by the expression $(36,39)$ :

$R_{\text {diff }}=\frac{\frac{K_{a}^{V}}{K_{a}^{G S T}}+\frac{[G S T]}{[V]}}{\frac{1}{k_{o f f}^{V}}\left(\frac{[G S T]}{[V]}\right)+\frac{1}{k_{o f f}^{G S}}\left(\frac{K_{a}^{V}}{K_{a}^{G S T}}\right)}$

where $K_{a}$ represents the association constant and $k_{\text {off }}$ the dissociation rate constant of bilirubin from GST or from vesicles, as indicated. At constant GST concentration, the collisional model (Eq. 1) predicts a direct linear correlation between the bilirubin transfer rate and the ac-
Collision-mediated Transport

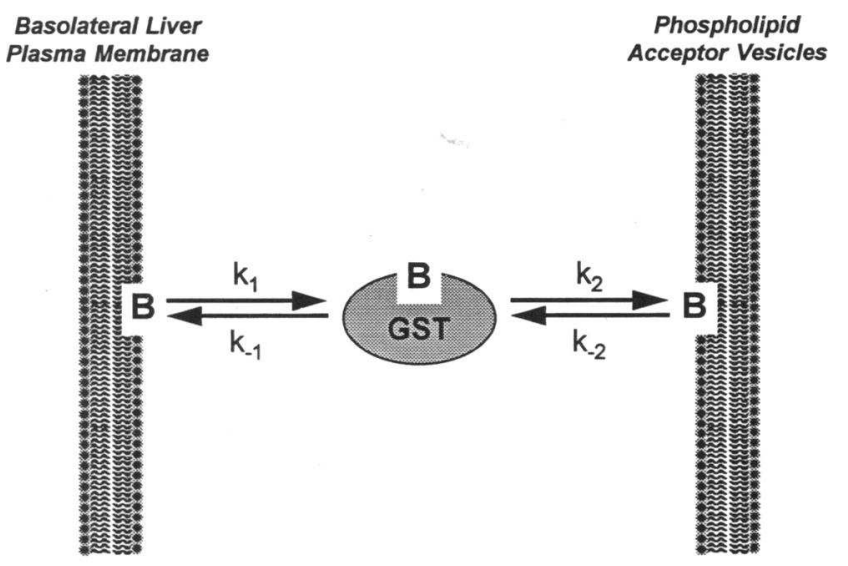

Diffusion-mediated Transport

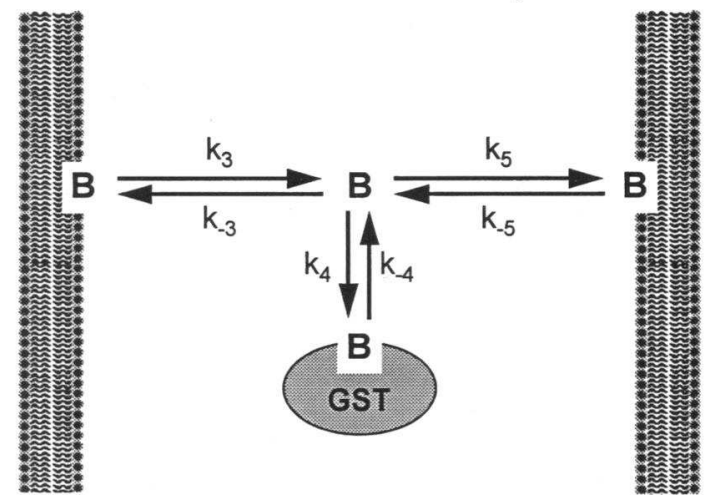

Figure 2. Kinetic models of GST-mediated bilirubin intermembrane transfer. This schematic diagram depicts kinetic models for the collisional and the diffusional transfer of bilirubin $(B)$ between basolateral plasma membranes and acceptor vesicles, in the presence of GST. The collisional model (top) assumes that bilirubin transfer occurs via direct interactions (collisions) between GST and the donor and acceptor membranes. Hence, GST functions as a bilirubin shuttle. The diffusional model (bottom) postulates that the interaction of bilirubin with GST or membranes occurs only after dissociation and aqueous diffusion of the bilirubin monomer. Rate constants are depicted by a $k$ with subscripted numerals.

ceptor vesicle concentration. In contrast, transfer occurring via a diffusional mechanism (Eq. 2) should asymptotically approach the GST dissociation rate as the number of acceptors is increased.

A three component system subsequently was used to determine the kinetics of GST-mediated bilirubin intermembrane transfer from rat liver basolateral plasma membranes to acceptor vesicles. The collisional and diffusional transfer models used in these analyses are detailed in Fig. 2. For collision-mediated transfer of bilirubin by glutathione S-transferase B, assuming that the concentration of the GST-bilirubin complex $([\mathrm{GST} \cdot \mathrm{B}])$ rapidly achieves steady state:

$$
\begin{aligned}
& \frac{d[G S T \cdot B]}{d t}=k_{1}[P M \cdot B][G S T]-k_{-1}[G S T \cdot B][P M] \\
& -\mathrm{k}_{2}[G S T \cdot B][V]+k_{-2}[V \cdot B][G S T]=0
\end{aligned}
$$

where $[P M \cdot B]$ and $[V \cdot B]$ are the concentrations of the plasma membrane-bilirubin and the vesicle-bilirubin complexes, and $[P M],[V]$, and $[G S T]$ are the concentrations of free plasma membranes, vesicles, 
and GST, respectively. The rate of appearance of bilirubin at the acceptor vesicle is given by the expression:

$\frac{d[V \cdot B]}{d t}=k_{2}[G S T \cdot B][V]-k_{-2}[V \cdot B][G S T]$.

Substituting for [GST $\cdot$ B ] using Eq. 3, under initial rate conditions (i.e., $[\mathrm{V} \cdot \mathrm{B}]=0)$, the bilirubin transfer rate is described by the equation (38):

$R_{\text {coll }}=\frac{k_{1} k_{2}[P M \cdot B][G S T]}{k_{-1}\left(\frac{[P M]}{[V]}\right)+k_{2}}$.

For a diffusional transfer process, if the bilirubin free monomer concentration [B] is assumed to reach steady state equilibrium rapidly, then:

$\frac{d[B]}{d t}=k_{3}[P M \cdot B]-k_{-3}[P M][B]-k_{4}[G S T][B]+$

$\mathbf{k}_{-4}[G S T \cdot B]-k_{5}[V][B]+k_{-5}[V \cdot B]=0$.

The rate of bilirubin arrival at the acceptor membranes can be described by the expression:

$\frac{d[V \cdot B]}{d t}=k_{5}[B][V]-k_{-5}[V \cdot B]$

Assuming initial rate conditions, where $[\mathrm{V} \cdot \mathrm{B}]$ and $[\mathrm{GST} \cdot \mathrm{B}]=0$, and substituting for [B] using Eq. 6, the rate of diffusion-mediated bilirubin transfer is given by $(38)$ :

$R_{\text {diff }}=\frac{k_{3} k_{5}[P M \cdot B]}{k_{-3}\left(\frac{[P M]}{[V]}\right)+k_{4}\left(\frac{[G S T]}{[V]}\right)+k_{5}}$.

If the concentrations of total bilirubin, plasma membranes, and acceptor vesicles are maintained constant then, according to the collisional model (Eq. 5), the initial rate of bilirubin transfer can be expressed as a linear function of the free GST concentration:

$R_{\text {coll }}=P_{1}[G S T]$

whereas the diffusional model (Eq. 8) reduces to:

$R_{\text {diff }}=\frac{P_{2}}{[G S T]+P_{3}}$

where $P_{1}, P_{2}$, and $P_{3}$ are constants. Thus, under the above conditions, a plot of the bilirubin transfer rate versus the concentration of glutathione S-transferase B is predicted to rise linearly for a collision-mediated transfer mechanism, whereas the rate of a diffusional process should decrease asymptotically with increasing GST levels.

\section{Results}

Unconjugated bilirubin quenches the steady state fluorescence of rat liver glutathione S-transferase B in a concentration-dependent manner $(5,8,10)$. The decrease in GST fluorescence induced by bilirubin binding was linear up to a 1:1 bilirubin/ protein molar ratio; hence, a ratio of bilirubin to GST below unity was used in all experiments.

Kinetic analysis of bilirubin transfer from glutathione $S$ transferase $B$ to acceptor vesicles. The spontaneous transfer of unconjugated bilirubin from rat liver glutathione S-transferase B to unlabeled small unilamellar phosphatidylcholine acceptor vesicles was monitored over time using stopped-flow fluorome-

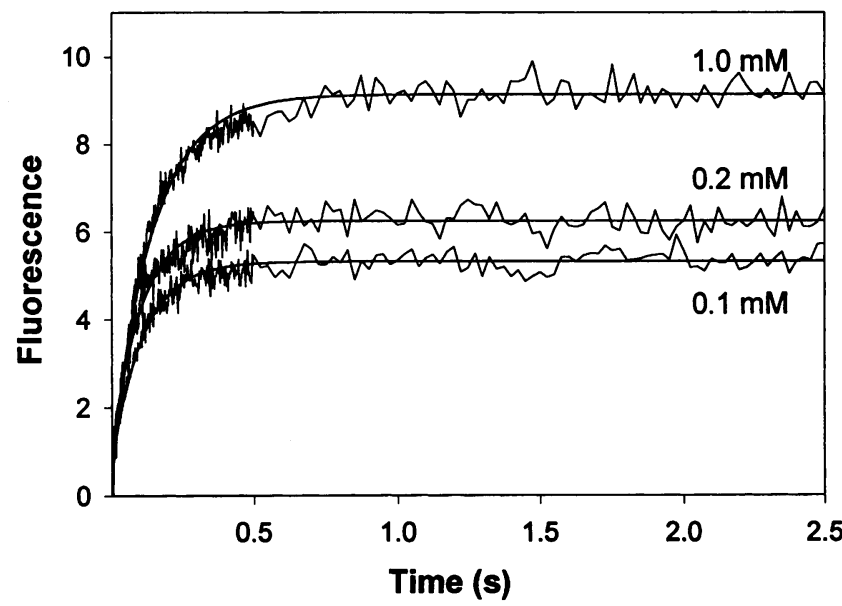

Figure 3. Transfer of bilirubin from glutathione S-transferase B to small unilamellar acceptor vesicles. Data from a series of experiments monitoring the spontaneous transfer of bilirubin from glutathione S-transferase B to small unilamellar phosphatidylcholine vesicles are presented. The tryptophan fluorescence of GST (ex: $280 \mathrm{~nm}$, em: $360 \mathrm{~nm}$ longpass filter) was monitored over a 2.5 -s interval, over which time steady state was achieved. The time-dependent reemergence of fluorescence reflects the transfer of unconjugated bilirubin $(1.0 \mu \mathrm{M})$ from an equimolar concentration of GST to increasing concentrations of acceptor vesicles (0.1-1.0 mM phospholipid). Each curve represents the mean of eight to ten repetitive stopped-flow injections performed at $25^{\circ} \mathrm{C}$ and is fitted by a single exponential function of the form $F(t)=a\left(1-e^{-k t}\right)$, as indicated by the solid line $(P<0.0005)$. The best-fit rate constants $(k)$ for the depicted curves were $9.1 \pm 0.2,9.5 \pm 0.3$, and $6.8 \pm 0.1 \mathrm{~s}^{-1}$ $( \pm \mathrm{SE}$ ) at $0.1,0.2$, and $1.0 \mathrm{mM}$ acceptor phospholipid. The corresponding amplitudes $(a)$ were $-4.7 \pm 0.1,-5.4 \pm 0.1$, and $-8.4 \pm 0.1$, respectively.

try (Fig. 3). Regression analysis of the fluorescence curves indicates that the transfer process is well-described by a single exponential function, with no significant improvement in fit obtained using a double exponential equation. The first-order transfer kinetics with respect to acceptor vesicle concentration provides support for the presence of a single, high-affinity bilirubin binding site on glutathione S-transferase B $(3,8)$. In Fig. 4 , the rates obtained from single exponential fits of the transfer curves are plotted against acceptor vesicle phospholipid concentration, for experiments performed at constant GST and bilirubin concentrations. The asymptotic decline in the bilirubin transfer rate with increasing acceptor vesicle concentration is inconsistent with a collision-mediated transfer process (Eq. 1), as an increase in the acceptor concentration causes a corresponding increase in the number of collisions per unit time and, therefore, should result in a more rapid rate. The data are entirely consistent with the diffusional transfer of bilirubin through the aqueous phase (Eq. 2). The mean rate constant for bilirubin transfer from GST to acceptor vesicles, obtained from the asymptote of the curve fit from Fig. 4, is $8.0 \pm 0.7 \mathrm{~s}^{-1}( \pm \mathrm{SD})$, corresponding to a halftime of $87 \mathrm{~ms}$.

Bilirubin dissociation from glutathione S-transferase B also was monitored by the time-dependent changes in dansyl-labeled $(0.2 \mathrm{~mol} \%)$ acceptor vesicle fluorescence. Transfer rates were identical to those obtained by measuring the tryptophan fluorescence of the protein, confirming that the observed fluorescence changes reflect the transfer of bilirubin from GST to acceptor vesicles and are not the result of conformational 


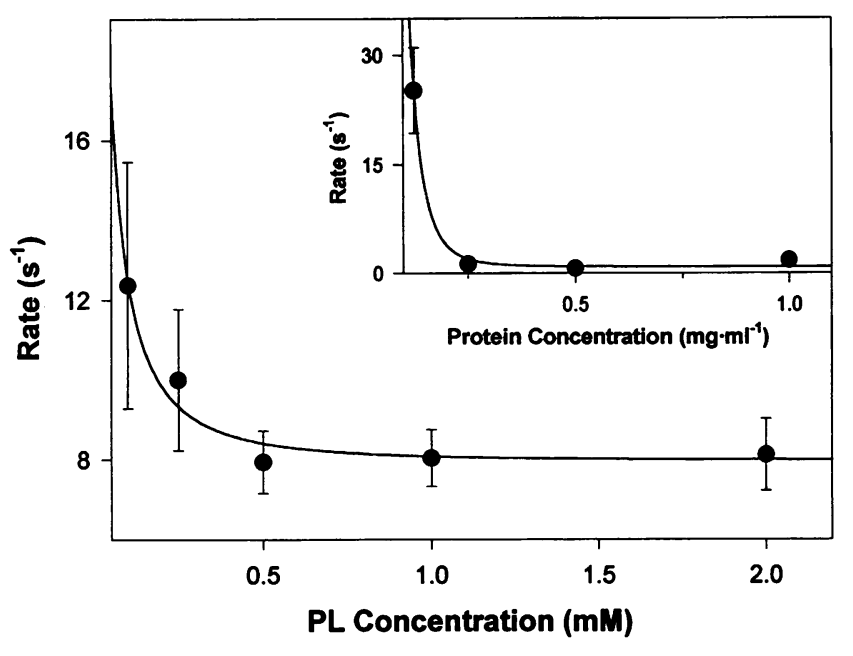

Figure 4. Effect of acceptor vesicle concentration on the rate of bilirubin transfer from glutathione S-transferase B. The results of a series of experiments measuring the rate of bilirubin transfer from glutathione $S$ transferase B to small unilamellar phosphatidylcholine vesicles are displayed. The transfer rate is plotted versus the acceptor vesicle phospholipid concentration $(0.1-2.0 \mathrm{mM})$, with both the bilirubin and GST concentrations maintained at $1.0 \mu \mathrm{M}$. Each point represents the mean $\pm \mathrm{SD}$ of three separate sets of experiments performed at $25^{\circ} \mathrm{C}$. The solid line $(P<0.005)$ was generated from the best-fit parameters for a diffusional transfer mechanism (Eq. 2). The findings are inconsistent with the collisional model (Eq. 1), which predicts a linear increase in transfer rate. The inset summarizes data obtained for bilirubin $(1.0 \mu \mathrm{M})$ transfer from GST $(1.0 \mu \mathrm{M})$ to fluorescent-labeled rat liver microsomes $(0.13-1.0 \mu \mathrm{g}$ protein $/ \mathrm{ml})$. In these studies, transfer was monitored by the time-dependent increase in rhodamine fluorescence (ex: $450 \mathrm{~nm}$, em: $535 \mathrm{~nm}$ long-pass filter). As observed with model acceptor vesicles, these results are consistent with a diffusional process (solid line, $P$ $<0.0005$ ).

changes in the GST molecule. To examine whether the presence of microsomal proteins alters the mechanism of bilirubin transfer, the spontaneous delivery of bilirubin from GST to rhodamine-labeled rat liver microsomal membranes was studied. As was observed with model acceptor vesicles, at constant GST and bilirubin concentrations, an exponential decline in the transfer rate was observed as the number of microsomes was increased (Fig. 4, inset), indicative of a diffusional mechanism of bilirubin transfer. Collectively, these data support the concept that the transfer of bilirubin from GST to either model or native microsomal membrane vesicles occurs via aqueous diffusion.

The rate of bilirubin transfer from glutathione S-transferase B to small unilamellar phosphatidylcholine vesicles was measured over a range of temperatures $\left(18-40^{\circ} \mathrm{C}\right)$ to determine the thermodynamic activation parameters for bilirubin dissociation (26). An acceptor vesicle concentration $(1.0 \mathrm{mM})$ far in excess of GST $(1.0 \mu \mathrm{M})$ was selected so as to minimize reverse transfer of bilirubin and thereby to ensure that the measured rate solely reflects bilirubin dissociation from glutathione S-transferase B. From the slope of an Arrhenius plot (Fig. 5), the free energy of activation $\left(\Delta \mathrm{G}^{\ddagger}\right)$ at $25^{\circ} \mathrm{C}$ was calculated to be 16.4 $\mathrm{kcal} \cdot \mathrm{mol}^{-1}$, with enthalpic $\left(\Delta \mathrm{H}^{\ddagger}\right)$ and entropic $\left(\mathrm{T} \Delta \mathrm{S}^{\ddagger}\right)$ contributions of 10.0 and $-6.4 \mathrm{kcal} \cdot \mathrm{mol}^{-1}$, respectively. These thermodynamic parameters are similar to those obtained for bilirubin dissociation from human serum albumin which, as reported

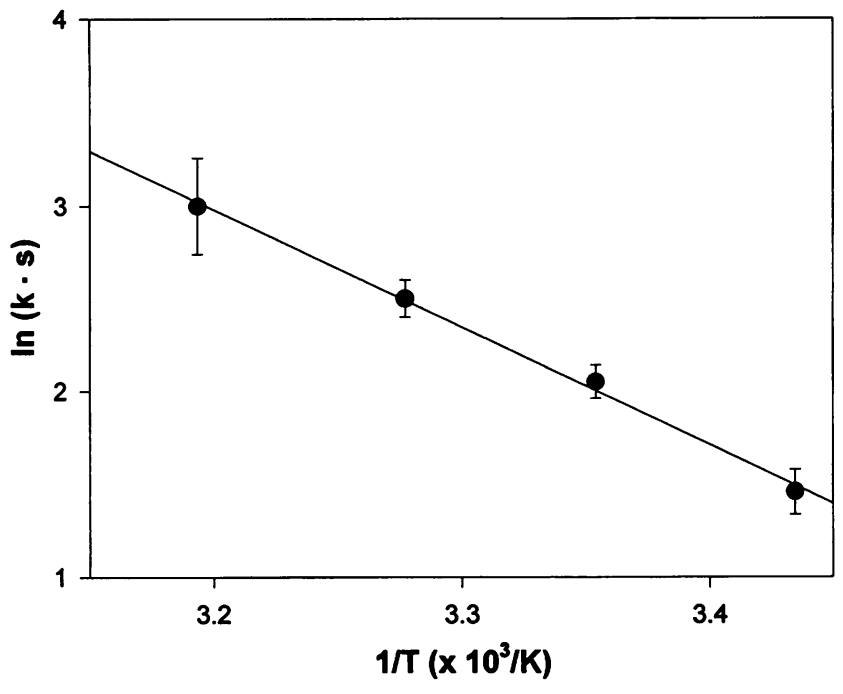

Figure 5. Arrhenius plot of bilirubin dissociation from glutathione Stransferase B. The rate of bilirubin transfer from rat liver glutathione S-transferase B to small unilamellar phosphatidylcholine vesicles was measured over a temperature range of $18-40^{\circ} \mathrm{C}$ by changes in GST fluorescence. The concentrations of GST, bilirubin, and vesicle phospholipid were $1.0 \mu \mathrm{M}, 1.0 \mu \mathrm{M}$, and $1.0 \mathrm{mM}$, respectively. Each point represents the mean $\pm S D$ of three separate sets of experiments. The natural $\log$ of the transfer rate constant $(k)$ is plotted against inverse temperature $\left(K^{-1}\right)$, and the activation energies for the dissociation of bilirubin from GST were determined from the slope of the linear fit ( $P$ $<0.0005$ ).

previously (36), exhibits a $\Delta \mathrm{G}^{\ddagger}$ of $17.3, \Delta \mathrm{H}^{\ddagger}$ of 12.1 , and $\mathrm{T} \Delta \mathrm{S}^{\ddagger}$ of $-5.2 \mathrm{kcal} \cdot \mathrm{mol}^{-1}$.

Kinetics of bilirubin transfer from phospholipid vesicles to GST. The spontaneous rate of bilirubin transfer from dansyllabeled $(0.5 \mathrm{~mol} \%)$ small unilamellar phosphatidylcholine vesicles to GST was measured by the reemergence of donor vesicle fluorescence over time. To obtain a broad range of acceptor concentrations, the quantities of GST required for this study were higher than those attainable with pure glutathione S-transferase B. Hence, these studies used unfractionated rat liver GST, which consists primarily of alpha class $(65-85 \%)$ and, to a lesser extent, $m u$ and $p i$ classes of GST $(15,44)$. Since the GST alpha class (YaYc) binds bilirubin with significantly higher affinity than the $m u$ and $p i$ classes $(8,9)$, glutathione Stransferase $B$ is presumed to be serving as the principal acceptor protein in these studies, as supported by experiments demonstrating that the rate of bilirubin transfer from unfractionated GST to acceptor vesicles is virtually identical to that obtained with similar concentrations of purified glutathione S-transferase B (data not shown). A plot of the bilirubin transfer rate versus the donor vesicle phospholipid/GST molar ratio (Fig. 6) exhibits an asymptotic decline, consistent with a diffusional process (Eq. 2). Indeed, as the relative concentration of GST to donor vesicles is increased, the rate of bilirubin transfer rises toward that previously reported for bilirubin dissociation from small unilamellar vesicles (26). Thus, it appears that bilirubin delivery to, and dissociation from, GST occurs via a diffusional mechanism.

Bilirubin transfer from basolateral liver plasma membranes to small unilamellar phosphatidylcholine vesicles in the presence of glutathione S-transferase B. The rate of bilirubin trans- 


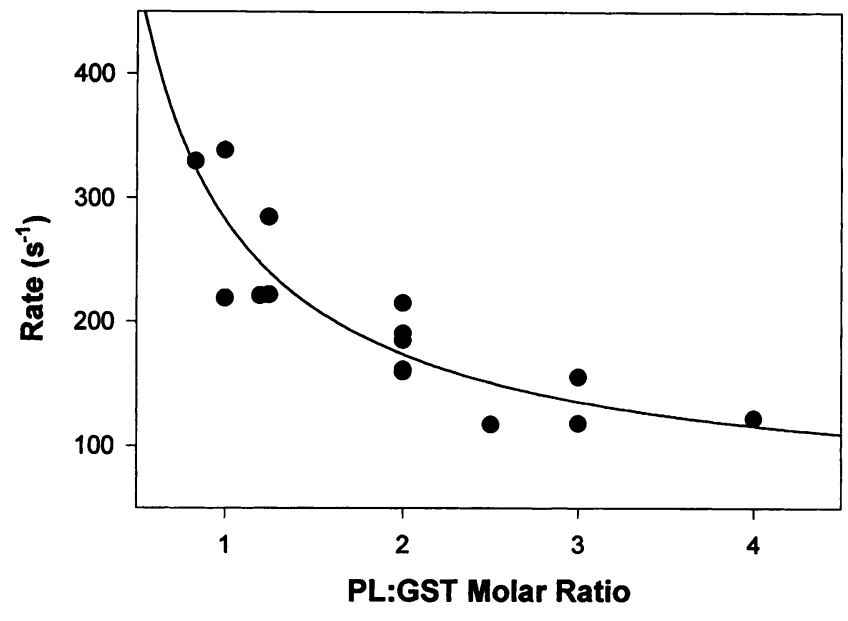

Figure 6. Bilirubin transfer from phospholipid vesicles to hepatic GST: influence of the donor/acceptor molar ratio. The rate of bilirubin transfer from dansyl-labeled $(0.5 \mathrm{~mol} \%)$ small unilamellar phosphatidylcholine vesicles (20-50 $\mu \mathrm{M}$ phospholipid) to unfractionated rat liver GST (5$50 \mu \mathrm{M}$ ) was determined by monitoring the time-dependent changes in dansyl fluorescence. The molar ratio of bilirubin/donor vesicle phospholipid was 1:200 for all experiments, which were performed at $25^{\circ} \mathrm{C}$. The first-order transfer rate constant is plotted versus the donor vesicle phospholipid/GST molar ratio, with each point representing the average of $5-10$ stopped-flow injections. The solid line $(P<0.0005)$ was generated from best-fit parameters for the diffusional model of bilirubin transfer (Eq. 2).

fer from rat liver basolateral plasma membrane donor vesicles to dansyl-labeled small unilamellar phosphatidylcholine acceptor vesicles was analyzed both in the presence and absence of glutathione S-transferase B to characterize the effect of GST on the kinetics of bilirubin intermembrane movement. The data from a representative set of transfer experiments are displayed in Fig. 7. In these studies, the concentration of acceptor vesicles was in large excess of donor vesicles to minimize the contribution of reverse bilirubin transfer to the overall rate (i.e., initial rate conditions ). At constant bilirubin, plasma membrane, and phospholipid vesicle concentrations, the rate of bilirubin delivery to acceptor vesicles declined asymptotically with increasing GST concentration (Fig. 8). These results cannot be explained on the basis of bilirubin redistribution to GST since, given the marked excess of donor and acceptor vesicles, GST binds a trivial percentage of the total bilirubin present $(<5 \%$ at the highest GST concentrations used). The findings are consistent with a diffusional mechanism of bilirubin transfer (Eq. 10) and are incompatible with collision-mediated transfer (Eq. 9), as the latter model predicts a linear increase in rate. It should be noted that even when reverse transfer of ligand from acceptor vesicles is considered (i.e., non-initial rate conditions), the results wholly support a diffusional, as opposed to a collisional transfer mechanism. In summary, these data, which demonstrate a decreasing bilirubin intermembrane transfer rate with increasing concentrations of glutathione S-transferase B, suggest that GST does not function as an intracellular transport protein for bilirubin.

\section{Discussion}

The liver is responsible for the uptake, metabolism, and biliary excretion of a variety of small hydrophobic and amphipathic

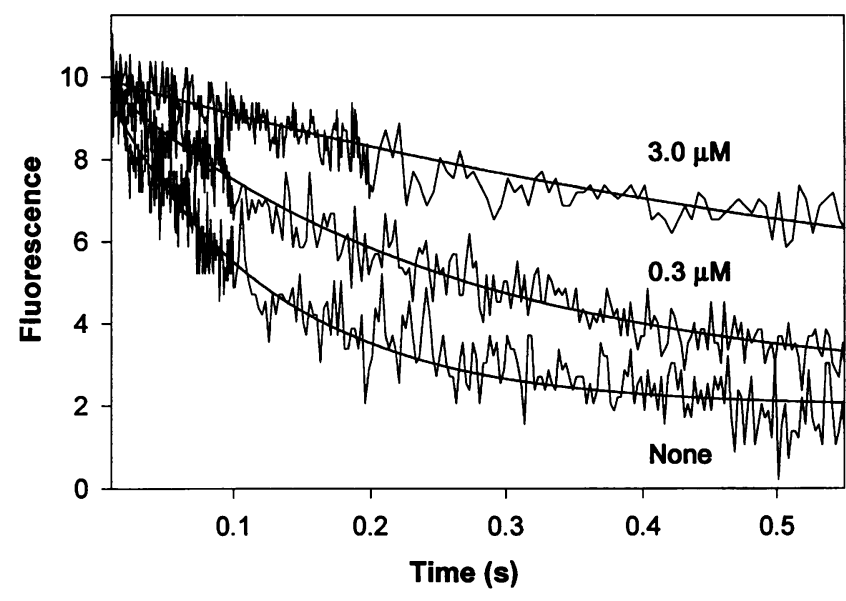

Figure 7. Influence of glutathione S-transferase B on bilirubin transfer from basolateral plasma membranes to phosphatidylcholine acceptor vesicles. Fluorescence recordings of bilirubin $(0.5 \mu \mathrm{M})$ transfer from rat basolateral liver plasma membranes $(250 \mu \mathrm{g}$ protein $/ \mathrm{ml})$ to dansyllabeled $(0.2 \mathrm{~mol} \%)$ small unilamellar phosphatidylcholine vesicles $(0.5$ $\mathrm{mM}$ phospholipid) in the presence of increasing concentrations of glutathione S-transferase B are displayed. The time-dependent decrease in fluorescence intensity is due to quenching of the dansyl probe on bilirubin binding to the acceptor vesicles. Each curve, which is normalized to a scale of $0-10$, represents the mean of six to eight repetitive injections performed at $25^{\circ} \mathrm{C}$ and is well-described by a single-exponential function (solid line, $P<0.0005$ ). The best-fit rate constants for the depicted curves were $8.3 \pm 0.3,4.1 \pm 0.2$, and $1.5 \pm 0.1 \mathrm{~s}^{-1}( \pm \mathrm{SE})$ in the presence of $0,0.3 \mu \mathrm{M}$, and $3.0 \mu \mathrm{M}$ GST. The corresponding amplitudes were $8.0 \pm 0.1,7.5 \pm 0.1$, and $6.6 \pm 0.3$, respectively. The rate of bilirubin transfer is observed to decline with increasing GST concentration.

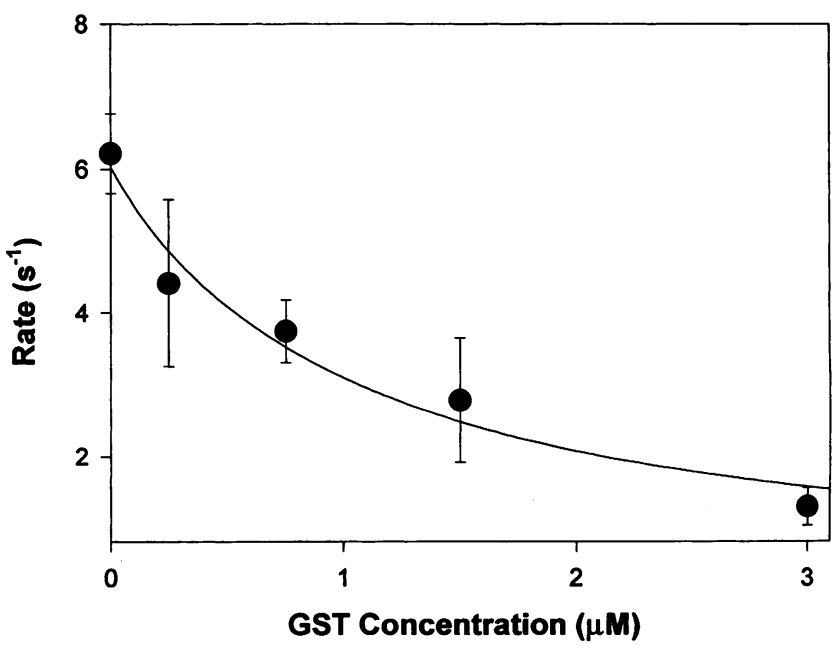

Figure 8. Effect of glutathione S-transferase B on bilirubin intermembrane transfer. The rates of bilirubin transfer from isolated rat basolateral liver plasma membranes to dansyl-labeled $(0.2 \mathrm{~mol} \%)$ small unilamellar phosphatidylcholine acceptor vesicles at $25^{\circ} \mathrm{C}$ are plotted against the concentration of glutathione S-transferase B. The concentrations of bilirubin $(0.5 \mu \mathrm{M})$, bLPM $(250 \mu \mathrm{g}$ protein $/ \mathrm{ml})$, and vesicle phospholipid $(0.5 \mathrm{mM})$ were similar for all experiments. The bilirubin transfer rate declines asymptotically with increasing GST concentration (solid line, $P<0.001$ ), as predicted by the diffusional model (Eq. 10). 
compounds, including unconjugated bilirubin. After uptake from plasma, bilirubin is transported from the hepatocyte sinusoidal ( basolateral) plasma membrane to the endoplasmic reticulum, where the molecule undergoes glucuronidation, a reaction catalyzed by the microsomal enzyme bilirubin UDP-glucuronosyltransferase. The resultant bilirubin glucuronides are secreted into the canalicular lumen by the multispecific organic anion transporter located on the canalicular (apical) surface. Because of the poor aqueous solubility of unconjugated bilirubin, it generally has been presumed that the intracellular transport of this compound is mediated by cytosolic binding proteins, with glutathione S-transferase B representing the principal candidate bilirubin transporter in the liver $(1,4,13,16)$ and other tissues $(2$, $6,45-47)$. However, there has been little direct evidence to support a role for GST in the transport of bilirubin, and other nonsubstrate ligands, within the cell. In fact, we have reported previously that, in the absence of soluble proteins, the spontaneous transfer of bilirubin between model and native membrane vesicles occurs by rapid diffusion of monomeric bilirubin through the aqueous phase $(26,27)$. These observations raise important questions about the vital role of GST in cellular transport processes.

The current investigations indicate that the spontaneous transfer of bilirubin between glutathione S-transferase B and both model and isolated native hepatocyte membranes occurs via aqueous diffusion and not by a collisional mechanism. These findings are in accordance with those of Boyer et al. (48), who demonstrated that GST have access to CDNB and sulfobromophthalein only when these ligands are present in the aqueous phase. The rate constant for bilirubin dissociation from glutathione S-transferase B is over 25 -fold slower than from small unilamellar phosphatidylcholine vesicles (26). While bilirubin dissociation from isolated rat liver plasma membranes is less rapid than from model vesicles (because of the larger diameter of the native membranes), the off-rate is $\sim 1.5-3$-fold faster than from GST (27). Moreover, the diffusion coefficient for the bilirubin monomer likely exceeds that of the bilirubinGST complex by at least one order of magnitude (13). Taken together, these findings suggest that direct intermembrane transfer of monomeric bilirubin through the aqueous phase is significantly more rapid than GST-mediated bilirubin transport. Hence, it would appear unlikely that glutathione S-transferase B serves an intracellular transport function, at least with regard to unconjugated bilirubin.

We further examined this hypothesis by directly measuring the rate of intermembrane bilirubin transfer between native hepatocyte basolateral plasma membranes and phospholipid acceptor vesicles in the presence of glutathione S-transferase B. Model phosphatidylcholine vesicles, rather than labeled microsomal membranes, were used as acceptors to enhance the sensitivity of the system. The validity of this strategy is based on our observation that the kinetics of bilirubin transfer from glutathione S-transferase B to phospholipid vesicles is similar to those for transfer to isolated microsomes, suggesting that integral microsomal proteins play little role in ligand dissociation from GST or binding to acceptor membranes (27). As predicted by the diffusional model (Eq. 10), increasing GST concentrations resulted in a reduced rate of bilirubin delivery to acceptor membranes. With the sole exception of liver fatty acid binding protein (FABP) $(49,50)$, the majority of cytosolic transport proteins, including heart FABP (43), adipocyte FABP (41), nonspecific lipid transfer protein $(38,40,51)$, and phosphati- dylcholine transfer protein (42), exhibit collisional transport kinetics. It also is notable that each of these proteins, including liver FABP (52), has been shown to enhance the intermembrane transfer rate of the corresponding ligands. In contrast, glutathione S-transferase B does not increase, but rather slows, the rate of bilirubin intermembrane movement. Hence, contrary to the kinetic behavior typical of intracellular transport proteins, increased concentrations of GST would appear to result in a slower rate of ligand delivery from the plasma membrane to the endoplasmic reticulum.

The data presented in Fig. 8 indicate that varying the concentration of GST between 0.25 and $3 \mu \mathrm{M}$ results in a fivefold change in the rate of bilirubin intermembrane transfer. Since the diffusional transfer model (Eq. 8) predicts that it is the molar ratio of GST to acceptor vesicles which is the key determinate of the overall rate of bilirubin transfer, these results can reasonably be extrapolated to in vivo events. The intracellular concentration of GST in the hepatocyte is $\sim 10 \mu \mathrm{M}(0.45 \mathrm{mg} / \mathrm{ml})(15)$, and the phospholipid concentration of the endoplasmic reticulum is estimated at $10-40 \mathrm{mM}$ (53). Assuming a physiologic hepatocellular GST/microsomal phospholipid molar ratio of 1:1,000, a doubling of the GST concentration would be predicted to result in a $25 \%$ reduction in cellular transit time, while a halving of intracellular GST levels would enhance bilirubin movement through the cytosol by $\sim 20 \%$. If delivery to the endoplasmic reticulum constitutes a key rate-limiting step in bilirubin conjugation by the hepatocyte, then GST may represent an important control point for bilirubin metabolism. Indirect support for this concept comes from data demonstrating that the rate of bilirubin glucuronide formation by isolated hepatic microsomes is modulated by the concentration and type of bilirubin donor (54).

The physiologic relevance of nonsubstrate ligand binding to GST remains conjectural. There is evidence that the interaction of various endogenous compounds (e.g., bilirubin, heme, corticosterone, triiodothyronine) and xenobiotics (e.g., hematin, sulfobromophthalein, cephalothin, morphine sulfate) with GST, at a location independent of the active site, regulates enzymatic activity $(4,5,8,9,55,56)$, primarily as a result of induced changes in protein conformation (57). Thus, the high-affinity interaction of nonsubstrate ligands with GST may represent an epiphenomenon of binding at this secondary binding site. Alternatively, it has been proposed that the GST function as an intracellular binding reservoir $(2,58)$. In this capacity, it is postulated that GST serve a cytoprotective role by decreasing the partitioning of hydrophobic and amphipathic molecules into cellular membranes. Other investigators have shown that the presence of high intracellular concentrations of GST facilitates net uptake of nonsubstrate ligands into the hepatocyte by decreasing ligand efflux from the cell $(1,12,59,60)$, although these observations have been challenged (61). It is notable that various xenobiotic compounds have been demonstrated to modulate GST gene expression in a host of tissues, including the liver $(44,62,63)$. Specific evidence in support of the potential regulation of GST expression by bilirubin is derived from the finding that erythrocytes of infants with neonatal jaundice (unconjugated hyperbilirubinemia) exhibit markedly elevated GST activity as compared with nonjaundiced newborns (64). In light of these observations, our data raise the intriguing possibility that the hepatocyte (and potentially other epithelial cells) may regulate the delivery of nonsubstrate ligands to intracellular sites of metabolism by modulating the cytosolic levels of GST. 


\section{Acknowledgments}

The authors gratefully acknowledge Drs. Mark Zeidel, Carey Waldburger, and Robert Sauer for their kind advice and support with the performance of stopped-flow experiments and Ms. Emma Bootle for her technical assistance.

This study was supported by National Institutes of Health Research Grants DK-02047, DK-36887, DK-43955, and DK-34854, a Harvard Digestive Diseases Center Pilot/Feasibility Award (to S. D. Zucker), an American Liver Foundation Student Research Award (to W. Goessling), and by the Alfried Krupp von Bohlen und Halbach-Stiftung Scholarship Foundation (to W. Goessling).

\section{References}

1. Kaplowitz, N. 1980. Physiological significance of glutathione S-transferases. Am. J. Physiol. 239:G439-G444.

2. Boyer, T. D. 1989. The glutathione S-transferases: an update. Hepatology 9:486-496.

3. Kamisaka, K., I. Listowsky, Z. Gatmaitan, and I. M. Arias. 1975. Interactions of bilirubin and other ligands with ligandin. Biochemistry. 14:2175-2180.

4. Tipping, E., B. Ketterer, L. Christodoulides, and G. Enderby. 1976. The non-covalent binding of small molecules by ligandin: interactions with steroids and their conjugates, fatty acids, bromosulphophthalein, carcinogens, glutathione and related compounds. Eur. J. Biochem. 67:583-590.

5. Ketley, J. N., W. H. Habig, and W. B. Jakoby. 1975. Binding of nonsubstrate ligands to the glutathione S-transferases. J. Biol. Chem. 250:8670-8673.

6. Listowsky, I., M. Abramovitz, H. Homma, and Y. Niitsu. 1988. Intracellular binding and transport of hormones and xenobiotics by glutathione S-transferases. Drug Metab. Rev. 19:305-318.

7. Kamisaka, K., W. H. Habig, J. N. Ketley, I. M. Arias, and W. B. Jakoby 1975. Multiple forms of human glutathione S-transferase and their affinity for bilirubin. Eur. J. Biochem. 60:153-161.

8. Vander Jagt, D. L., S. P. Wilson, V. L. Dean, and P. C. Simons. 1982. Bilirubin binding to rat liver ligandins (glutathione S-transferases A and B) relationship between bilirubin binding and transferase activity. J. Biol. Chem. 257:1997-2001.

9. Sugiyama, Y., M. Sugimoto, A. Stolz, and N. Kaplowitz. 1984. Comparison of the binding affinities of five forms of rat glutathione S-transferases for bilirubin, sulfobromophthalein and hematin. Biochem. Pharmacol. 33:3511-3513.

10. Simons, P. C., and D. L. Vander Jagt. 1980. Bilirubin binding to human liver ligandin (glutathione S-transferase). J. Biol. Chem. 255:4740-4744.

11. Vos, R. M. E., and P. J. van Bladeren. 1990. Glutathione S-transferases in relation to their role in the biotransformation of xenobiotics. Chem. Biol. Interact. 75:241-265.

12. Levi, A. J., Z. Gatmaitan, and I. M. Arias. 1969. Two hepatic cytoplasmic protein fractions, $\mathrm{Y}$ and $\mathrm{Z}$, and their possible role in the hepatic uptake of bilirubin sulfobromophthalein, and other anions. J. Clin. Invest. 48:2156-2167.

13. Tipping, E., and B. Ketterer. 1981. The influence of soluble binding proteins on lipophile transport and metabolism in hepatocytes. Biochem. $J$. 195:441-452.

14. Fleischner, G., J. Robbins, and I. M. Arias. 1972. Immunologic studies of $\mathrm{Y}$ protein: a major cytoplasmic organic anion-binding protein in rat liver. $J$. Clin. Invest. 51:677-684.

15. van Ommen, B., J. J. P. Bogaards, W. H. M. Peters, B. Blaauboer, and P. J. van Bladeren. 1990. Quantification of human hepatic glutathione S-transferase. Biochem. J. 269:609-613.

16. LeBlanc, G. A. 1994. Hepatic vectorial transport of xenobiotics. Chem. Biol. Interact. 90:101-120

17. Boyer, T. D., and E. Olsen. 1991. Role of glutathione S-transferases in heme transport. Biochem. Pharmacol. 42:188-190.

18. Liem, H. H., J. A. Grasso, S. H. Vincent, and U. Muller-Eberhard. 1990. Protein-mediated efflux of heme from isolated rat liver mitochondria. Biochem. Biophys. Res. Commun. 167:528-534.

19. Senjo, M., T. Ishibashi, and Y. Imai. 1985. Purification and characterization of cytosolic liver protein facilitating heme transport into apocytochrome b5 from mitochondria: evidence for identifying the heme transfer protein as belonging to a group of glutathione S-transferases. J. Biol. Chem. 260:9191-9196.

20. Husby, P., K. Srai, B. Ketterer, and I. Romslo. 1981. Effect of ligandin on the efflux of co-deuteroporphyrin from isolated rat liver mitochondria. Biochem. Biophys. Res. Commun. 100:651-659.

21. Bennett, C. F., and L. C. Yeoman. 1987. Microinjected glutathione Stransferase $\mathrm{Yb}$ subunits translocate to the cell nucleus. Biochem. J. 247:109-112.

22. Trotter, P. J., and D. R. Voelker. 1994. Lipid transport processes in eukaryotic cells. Biochim. Biophys. Acta. 1213:241-262.

23. Wirtz, K. W. A. 1991. Phospholipid transfer proteins. Annu. Rev. Biochem. 60:73-99.
24. Mastro, A. M., and A. D. Keith. 1984. Diffusion in the aqueous compartment. J. Cell Biol. 99:180-187.

25. Luby-Phelps, K., P. E. Castle, D. L. Taylor, and F. Lanni. 1987. Hindered diffusion of inert tracer particles in the cytoplasm of mouse 3T3 cells. Proc. Natl. Acad. Sci. USA. 84:4910-4913.

26. Zucker, S. D., J. Storch, M. L. Zeidel, and J. L. Gollan. 1992. Mechanism of the spontaneous transfer of unconjugated bilirubin between small unilamellar phosphatidylcholine vesicles. Biochemistry. 31:3184-3192.

27. Zucker, S. D., W. Goessling, M. L. Zeidel, and J. L. Gollan. 1994. Membrane lipid composition and vesicle size modulate bilirubin intermembrane transfer: evidence for membrane-directed trafficking of bilirubin in the hepatocyte. $J$. Biol. Chem. 269:19262-19270.

28. Spivak, W., and W. Yuey. 1986. Application of a rapid and efficient h.p.l.c. method to measure bilirubin and its conjugates from native bile and in model bile systems: potential use as a tool for kinetic reactions and as an aid in diagnosis of hepatobiliary disease. Biochem. J. 234:101-109.

29. Habig, W. H., and W. B. Jakoby. 1981. Assays for differentiation of glutathione S-transferases. Methods Enzymol. 77:398-405.

30. Barenholz, Y., D. Gibbes, B. J. Litman, J. Goll, T. E. Thompson, and F. D. Carlson. 1977. A simple method for the preparation of homogeneous phospholipid vesicles. Biochemistry. 16:2806-2810.

31. Bartlett, G. R. 1959. Phosphorus assay in column chromatography. $J$. Biol. Chem. 234:466-468.

32. Stubbs, C. D., K. Kinosita, F. Munkonge, P. J. Quinn, and A. Ikegami 1984. The dynamics of lipid motion in sarcoplasmic reticulum membranes determined by steady-state and time-resolved fluorescence measurements on 1,6-diphenyl-1,3,5-hexatriene and related molecules. Biochim. Biophys. Acta. 775:374380 .

33. Meier, P. J., and J. L. Boyer. 1990. Preparation of basolateral (sinusoidal) and canalicular plasma membrane vesicles for the study of hepatic transport processes. Methods Enzymol. 192:534-545.

34. Scharschmidt, B. F., E. B. Keeffe, N. M. Blankenship, and R. K. Ockner. 1979. Validation of a recording spectrophotometric method for measurement of membrane associated $\mathrm{Mg}^{++}$and $\mathrm{Na}^{+} \mathrm{K}^{+}$-ATPase activity. J. Lab. Clin. Med. 93:790-799.

35. Hahm, J. S., J. D. Ostrow, P. Mukerjee, and L. Celic. 1992. Ionization and self-association of unconjugated bilirubin, determined by rapid solvent partition from chloroform, with further studies of bilirubin solubility. J. Lipid Res. 33:1123-1137.

36. Zucker, S. D., W. Goessling, and J. L. Gollan. 1995. Kinetics of bilirubin transfer between serum albumin and membrane vesicles: insight into the mechanisms of organic anion delivery to the hepatocyte plasma membrane. J. Biol. Chem. 270:1074-1081

37. Cu, A., G. G. Bellah, and D. A. Lightner. 1975. On the fluorescence of bilirubin. J. Am. Chem. Soc. 97:2579-2580.

38. Nichols, J. W. 1988. Kinetics of fluorescent-labeled phosphatidylcholine transfer between nonspecific lipid transfer protein and phospholipid vesicles. Biochemistry. 27:1889-1896.

39. Nichols, J. W., and R. E. Pagano. 1982. Use of resonance energy transfer to study the kinetics of amphiphile transfer between vesicles. Biochemistry. 21:1720-1726.

40. Gadella, T. W. J., and K. W. A. Wirtz. 1994. Phospholipid binding and transfer by the nonspecific lipid-transfer protein (sterol carrier protein 2): a kinetic model. Eur. J. Biochem. 220:1019-1028.

41. Wootan, M. G., D. A. Bernlohr, and J. Storch. 1993. Mechanism of fluorescent fatty acid transfer from adipocyte fatty acid binding protein to membranes. Biochemistry. 32:8622-8627.

42. Runquist, E. A., and G. M. Helmkamp. 1988. Effect of acceptor membrane phosphatidylcholine on the catalytic activity of bovine liver phosphatidylcholine transfer protein. Biochim. Biophys. Acta. 940:21-32.

43. Kim, H. K., and J. Storch. 1992. Mechanism of free fatty acid transfer from rat heart fatty acid-binding protein to phospholipid membranes: evidence for a collisional process. J. Biol. Chem. 267:20051-20056.

44. Pickett, C. B., and A. Y. H. Lu. 1989. Glutathione S-transferases: gene structure, regulation, and biological function. Annu. Rev. Biochem. 58:743-764.

45. Kirsch, R., G. Fleischner, K. Kamisaka, and I. M. Arias. 1975. Structural and functional studies of ligandin, a major renal organic anion binding protein. J. Clin. Invest. 55:1009-1019.

46. Bhargava, M. M., N. Ohmi, I. Listowsky, and I. M. Arias. 1980. Subunit composition, organic anion binding, catalytic and immunological properties of ligandin from rat testis. J. Biol. Chem. 255:724-727.

47. Caccuri, A. M., A. Aceto, F. Piemonte, C. Di Ilio, N. Rosato, and G. Federici. 1990. Interaction of hemin with placental glutathione transferase. Eur. J. Biochem. 189:493-497.

48. Boyer, T. D., D. Zakim, and D. A. Vessey. 1983. Do the soluble glutathione S-transferases have direct access to membrane-bound substrates? Biochem. Pharmacol. 32:29-35.

49. Kim, H. K., and J. Storch. 1992. Free fatty acid transfer from rat liver fatty acid-binding protein to phospholipid vesicles: effect of ligand and solution properties. J. Biol. Chem. 267:77-82. 
50. Storch, J., and N. M. Bass. 1990. Transfer of fluorescent fatty acids from liver and heart fatty acid-binding proteins to model membranes. J. Biol. Chem. 265:7827-7831.

51. Nichols, J. W., and R. E. Pagano. 1983. Resonance energy transfer assay of protein-mediated lipid transfer between vesicles. J. Biol. Chem. 258:53685371.

52. McCormack, M., and P. Brecher. 1987. Effect of liver fatty acid binding protein on fatty acid movement between liposomes and rat liver microsomes. Biochem. J. 244:717-723.

53. DePierre, J. W., G. Andersson, and G. Dallner. 1988. Endoplasmic reticulum and Golgi complex. In The Liver: Biology and Pathobiology. I. M. Arias, H. Popper, D. A. Shafritz, W. B. Jakoby, and D. Schachter, editors. Raven Press, New York. 165-188.

54. Whitmer, D. I., J. C. Ziurys, and J. L. Gollan. 1984. Hepatic microsoma glucuronidation of bilirubin in unilamellar liposomal membranes: implications for intracellular transport of lipophilic substrates. J. Biol. Chem. 259:11969-11975.

55. Boyer, T. D., D. A. Vessey, C. Holcomb, and N. Saley. 1984. Studies of the relationship between the catalytic activity and binding of non-substrate ligands by the glutathione S-transferases. Biochem. $J$. 217:179-185.

56. Vessey, D. A., and T. D. Boyer. 1988. Characterization of the activation of rat liver glutathione S-transferases by nonsubstrate ligands. Toxicol. Appl. Pharmacol. 93:275-280.

57. Nishihira, J., T. Ishibashi, M. Sakai, S. Tsuda, and K. Hikichi. 1993. Identification of the hydrophobic ligand-binding region in recombinant glutathione S-transferase $\mathbf{P}$ and its binding effect on the conformational state of the enzyme. Archiv. Biochem. Biophys. 302:128-133.
58. Tipping, E., B. Ketterer, and L. Christodoulides. 1979. Interactions of small molecules with phospholipid bilayers: binding to egg phosphatidylcholine of some organic anions (bromosulphophthalein, oestrone sulphate, haem and bilirubin) that bind to ligandin and aminoazo-dye-binding protein A. Biochem. J. 180:327-337.

59. Wolkoff, A. W., C. A. Goresky, J. Sellin, Z. Gatmaitan, and I. M. Arias 1979. Role of ligandin in transfer of bilirubin from plasma into liver. Am. J. Physiol. 236:E638-E648.

60. Levi, A. J., Z. Gatmaitan, and I. M. Arias. 1970. Deficiency of hepatic organic anion binding protein, impaired organic anion uptake by liver and "physiologic" jaundice in newborn monkeys. N. Engl. J. Med. 283:1136-1139.

61. Theilmann, L., Y. R. Stollman, I. M. Arias, and A. W. Wolkoff. 1984. Does Z-protein have a role in transport of bilirubin and bromosulfophthalein by isolated perfused rat liver? Hepatology. 4:923-926.

62. Parola, M., M. E. Biocca, G. Leonarduzzi, E. Albano, M. U. Dianzani, K. S. Gilmore, D. J. Meyer, B. Ketterer, T. F. Slater, and K. H. Cheeseman. 1993. Constitutive and inducible profile of glutathione S-transferase subunits in biliary epithelial cells and hepatocytes isolated from rat liver. Biochem. J. 291:641-647.

63. Rushmore, T. H., and C. B. Pickett. 1993. Glutathione S-transferases: structure, regulation, and therapeutic implications. J. Biol. Chem. 268:1147511478.

64. Carmagnol, F., P. M. Sinet, J. Rapin, and H. Jerome. 1981. Glutathione S-transferase of human red blood cells; assay values in normal subjects and in two pathological circumstances: hyperbilirubinemia and impaired renal function. Clin. Chim. Acta. 117:209-217. 